\title{
Enhanced Optical Coherence Vibration Tomography for Subnanoscale-Displacement-Resolution Calibration of Piezoelectric Actuators
}

\author{
Shuncong Zhong ${ }^{1,2^{*}}$, Qiukun Zhang ${ }^{2,3}$ \\ ${ }^{1}$ Department of Naval Architecture, Ocean and Marine Engineering, University of \\ Strathclyde, Henry Dyer Building, 100 Montrose Street, Glasgow G4 OLZ, United \\ Kingdom \\ ${ }^{2}$ Laboratory of Optics, Terahertz and Non-destructive Testing, School of Mechanical \\ Engineering and Automation, Fuzhou University,No.2 Campus Road, Fuzhou 350108, P. \\ R. China \\ ${ }^{3}$ Fujian Key Laboratory of Medical Instrument and Pharmaceutical Technology, Fuzhou \\ 350108, P.R. China
}

\section{ABSTRACT}

We report a subnanoscale-displacement-resolution optical coherence vibration tomography (SOCVT) system for real-time calibration of piezoelectric actuators. The calibrations of the actuators at nanoscale or microscale displacement ranges were performed by varying the input voltage over the entire range in the ascending and descending directions. The computational and experimental results demonstrated that the developed SOCVT could be used to characterize the dynamic hysteretic behaviour, nonlinear effect, and impulsive behaviour of piezoelectric actuators. The SOCVT technique is non-contact and non-invasive in nature, making it ideal for real-time and in situ ultra-precision calibration of piezoelectric actuators, which are widely used in active vibration control and nanopositioning.

Key Words: Enhanced Optical coherence vibration tomography; Real-time calibration; Piezoelectric actuators; Nanopositioning. 


\section{INTRODUCTION}

Optical coherence tomography (OCT) is a non-invasive and cross-sectional imaging technique that permits, for example, three-dimensional images of micrometre resolution to be obtained from within the retina [1,2]. Historically, OCT systems were developed and optimized for medical applications. However, due to their excellent sensitivity and spatial resolution, OCT systems have been increasingly used for nonmedical applications, including detection and characterization of subsurface Hertzian cracks in ceramic materials [3], in vivo thickness measurement of the sweat film layer [4], and non-destructive and quantitative characterization of pharmaceutical tablet coatings $[5,6]$. OCT is also used in deformation characterizations [7-10]. Targowski et al. [10] used OCT to track deformations in paintings on canvas caused by periodic humidity changes. Recently, an optical coherence vibration tomography (OCVT) system was reported, by which structural vibration monitoring and inner structure characterization can be characterized simultaneously [11]. Both the vibration amplitude and frequency were demonstrated to be quantified with an axial resolution of $1 \mu \mathrm{m}$; therefore, this system cannot be used for nanoscale dynamic characterization of PZT actuators. For nanoscale characterization, laser Doppler vibrometry $[12,13]$, holographic interferometry [14-16], and Speckle interferometry [17] exhibit high reliability and enable wideband, phase-resolved, single-point vibration measurements. For these interferometry techniques, the displacement is obtained from the phase change of the interferometric patterns, providing extremely high depth resolution (down to $10^{-12} \mathrm{~m}$ ). 
However, the detection range is usually limited to half the wavelength of the laser source due to the 2 mp-phase ambiguity [13]. The detection range can be extended beyond the half wavelength limit by using phase-shift modulation (or phase unwrapping) techniques at the cost of increased instrument complexity and decreased measurement accuracy (related to the inherent phase-shift error) [17]. In the present work, we report a sub-nanoscale-resolution optical coherence tomography (SOCVT) system for real-time calibration of piezoelectric actuators in which the amplitude of the FFT of the spectral interferogram was used instead of the phase information. Therefore, phase-shift modulation techniques are not required in the SOCVT system. SOCVT is an absolute displacement measurement method; as a result, SOCVT does not require phase-shift modulation or the integration of the velocity along the time axis, both of which are required for laser Doppler vibrometers to obtain the displacement information. The non-contact and non-invasive SOCVT system is a tool for subnanoscale-displacementresolution calibration of actuators at nanoscale or microscale displacement ranges.

\section{ENHANCED OPTICAL COHERENCE VIBRATION TOMOGRAPHY}

Fig. 1 shows a schematic diagram of the use of spectral-domain optical coherence tomography for piezoelectric actuator calibration. The light from a 50-W halogen lamp (a short-coherence white light source) is delivered into a Michelson interferometer using a biconvex lens. The light is then split into reference and sample beams using a beam splitter (50/50). Both the "object image" and the "reference image" are formed at the entrance slit of a broadband CCD-based spectrometer 
(USB2000+, Ocean Optics, USA). Interference occurs when both the "object image" and the "reference image" are spatially matched in size and orientation and their optical path lengths are matched within the coherence length of the light source. Note that the minimum integration time of the spectrometer is limited to $1 \mathrm{~ms}$; i.e., it could conduct 1000 measurements in one second. Due to this limitation of the spectrometer, this SOCVT setup can only measure low-frequency vibrations with a frequency of up to 250 $\mathrm{Hz}$. However, the frequency range could be easily increased to tens of $\mathrm{kHz}$ if a highperformance spectrometer is used. The sample used here is a PZT stack actuator (AE0203D04F, Thorlabs). The vibration amplitude and frequency of the PZT actuator were controlled by the signal generated from a signal generator (Agilent 33220) and amplified by a programmable DC power supply (Chroma 62000P). Our SOCVT setup here (in which a linear CCD is used) is different from the 2DOCVT setup we developed recently [18]. The 2DOCVT requires a high-speed camera with area CCD sensors, which enable measurement of the 2D beam-like structural vibration with a displacement resolution of $\sim 0.1 \mathrm{~nm}$. Due to its high speed, the highest sampling frequency of $\sim 40 \mathrm{kHz}$ for the 2DOCT could be achieved if the lowest resolution of the PCO-TECH high-speed CCD camera was used [18].

For a vibrating sample, the spectral interferogram of the interferometer of an optical coherence tomography system can be expressed as [11]

$$
\begin{aligned}
I(\lambda, t)= & I_{0}(\lambda) S_{r}^{2}+I_{0}(\lambda)\left(S_{s 0}(\lambda)^{2}+S_{s 1}(\lambda)^{2}\right)+2 I_{0}(\lambda) S_{r} S_{s 0}(\lambda) \cos [2 \pi \Delta l(t) / \lambda] \\
& +2 I_{0}(\lambda) S_{r} S_{s 1}(\lambda) \cos [2 \pi \Delta l(t) / \lambda+4 \pi d / \lambda]+2 I_{0}(\lambda) S_{s 0} S_{s 1}(\lambda) \cos (4 \pi d / \lambda)
\end{aligned}
$$


where $\lambda$ is the wavelength of light in air, $I_{0}(\lambda)=\sqrt{\frac{\varepsilon_{0}}{\mu_{0}}}\left|E_{i}(\lambda)\right|^{2}$ is the intensity of the incident light, $\varepsilon_{0}$ and $\mu_{0}$ are the vacuum permittivity and the vacuum permeability, $S_{r}$ is the reflection coefficient of a reference and is wavelength-independent, and $S_{s 0}(\lambda)$ and $S_{s 1}(\lambda)$ correspond to the surface reflection and the coating/core interface reflection of the sample, respectively, which are usually wavelength dependent for a layered sample. For simplicity, here, we assume that the sample has a single coating layer of thickness $d$ and that the contribution from the multiple reflections within the coating is negligible. $\Delta l(t)$ is the optical path length difference between the reference and sample arms, which is a function of $t$ for a vibrating sample. For a static sample, that is, when time $t$ in Eq. (1) is a constant, the system operates as a traditional optical coherence tomography system [2-6]. The fast Fourier transform (FFT) of the measured spectral interferogram provides both the surface position (i.e., $\Delta l(t))$ and the depth profile of the sample [11]. We previously reported an OCVT system by which the layer thickness of the vibrating structures can be characterized. Furthermore, the vibration frequency and amplitude (with an axial resolution of $1 \mu \mathrm{m}$ ) could be measured simultaneously. The vibration amplitude was simply based on the FFT results of the measured spectral interferogram.

However, due to signal leakage effects [19-21], the calculation of amplitude, phase and frequency from the FFT of the measured finite-length spectral interferogram is normally different from the real one. Therefore, the vibration amplitude measured by the previously reported OCVT system [11] is not a perfect result. Generally, applying windowing functions to the measured spectral interferogram is a common method to minimize the effect of leakage. 
However, theoretically, the maximum relative error of the amplitude estimation will be $36.4 \%$ and $15.3 \%$ when Rectangle and Hanning windows, respectively, are used to minimize leakage [20]; i.e., the results of FFT of the measured spectral interferogram are not precise if only windowing functions were used in the FFT computation. Therefore, the results of FFT of the measured spectral interferogram must be corrected and enhanced for higher precision. In our present work, the Hanning window was used in the FFT of a measured spectral interferogram, and then an energy-centroid discrete spectrum correction method [20] was used to precisely determine the signal parameters, such as frequency, amplitude, etc., thereby enhancing the vibration amplitude measurement with a subnanoscale-displacement resolution of better than $0.05 \mathrm{~nm}$, which is much higher than those of our OCVT [11] and 2DOCVT [18] systems reported previously. The detailed theory of the energy-centroid discrete spectrum correction method can be found in Ref [21].

In our SOCVT system, according to the theory of energy-centroid discrete spectrum correction method, the surface position ( $P$ ) of the PZT actuators to be characterized could be precisely determined as follows:

$$
P=\left[\begin{array}{llll}
p_{t 1} & p_{t 2} & \cdots & p_{t M}
\end{array}\right]^{T}
$$

where

$$
\left.p_{t i}=\sum_{j=-q}^{j=q} F I_{i j} \cdot\left(L_{i j}+i\right) / \sum_{j=-q}^{j=q} F I_{i j}\right) /\left(N \cdot \Delta f_{M}\right) \cdot c / n / 2, i=1, \ldots, M
$$

$M$ is the number of spectral interferograms. $p_{t i}$ is the precise determination of the surface position of the vibrating PZT actuator at time $t_{i}$. The surface displacement curve of the vibration PZT actuator can be obtained from $p_{t i}, i=1, \ldots, M$. In Equation (3), $F I_{i}$ is 
the FFT of the spectral interferogram measured at time $t_{i}(i=1, \ldots, M) ; \mathrm{II}_{M \mathrm{j}}$ is the peak value of the $j$-th spectral line of FFT of the spectral interferogram, where $L_{M j}$ is the corresponding number of spectral lines in the discrete spectrum; $N$ is the length of the spectral interferogram; $C$ is the velocity of light in air; $n$ is refractive index of air $(n=1)$; $\Delta f_{M}$ is the frequency interval, which is equal to $c\left(\lambda_{M}-\lambda_{M-1}\right) /\left(\lambda_{M-1} \lambda_{M}\right)$, where $\lambda_{M-1}$ and $\lambda_{M}$ are the appropriate wavelengths; $q$ is the number of spectral lines used for spectral correction. Here, we set $q=3$, i.e., three points in the spectrum were used in the spectrum correction computation of the TFT of the measured spectral interferogram.

To demonstrate the improved performance of the OVCT system using the spectrum correction method, a simulated spectral interferogram of the interferometer of an optical coherence tomography were computed using Equation (1) with a displacement amplitude of $\Delta l(t)=0.1 \mathrm{~nm}$. Fig. 2 (a), without using the energy-centroid discrete spectrum correction method, no displacement (solid line with amplitude of zero) is observed if the displacement is in subnanometre scale. However, if the FFT of the simulated spectral interferogram is corrected using the energy-centroid spectrum correction method, then the subnanometre-scale vibration displacement can be observed. As shown in Fig. 2 (a), the sine, sawtooth, and square waves with amplitudes of $0.1 \mathrm{~nm}$ are precisely measured. The simulated results demonstrated that the enhanced SOCVT method is a powerful tool for the determination of subnanoscale displacements or deformations. 


\section{EXPERIMENTAL RESULTS AND DISCUSSTIONS}

To verify the capability of the enhanced SOCVT measurement, the PZT actuators at nanoscale or microscale displacement ranges were calibrated by varying the input voltage on the whole range in the ascending and descending directions and measured by the developed SOCVT.

A $0.1-\mathrm{Hz}$ sine wave with a voltage amplitude of $18 \mathrm{~V}$ was used to drive a multilayer piezoelectric stack actuator. This sine wave voltage caused the surface of the PZT actuator to vibrate at a frequency of $0.1 \mathrm{~Hz}$ with an amplitude of 410 nanometres. Again, without using the energy-centroid spectrum correction method, the surface displacement of the vibrating PZT could not be accurately calculated due to the inherent shortcoming of the FFT (leakage effect), as shown by the dashed line in Fig. 2 (b). Similarly, this nanoscale harmonic vibration of the PZT stack actuator can be obtained accurately, as shown by the solid line in Fig. 2 (b).

For subnanoscale vibration measurement, $0.1-\mathrm{Hz}$ sine waves with voltage amplitudes of $200 \mathrm{mV}, 40 \mathrm{mV}$ and $10 \mathrm{mV}$ generated by a signal generator manufactured by Agilent were used to drive the PZT actuator, resulting in the surface of the PZT to vibrate at a frequency of $0.1 \mathrm{~Hz}$ with amplitudes of approximately $4.5 \mathrm{~nm}, 0.9 \mathrm{~nm}$ and $0.2 \mathrm{~nm}$, respectively. Fig. 2 (c) shows the three vibration curves obtained using the SOCVT system. The results demonstrated that such subnanoscale displacements could be accurately characterized by the developed SOCVT system. Therefore, the SOCVT is a powerful method for monitoring of sub-nanometre vibrations or deformations. Note here that the maximum measurable amplitude of the vibrations or deformations of the 
SOCVT system is limited by the spectrometer resolution $\delta \lambda$ and is given by $z_{\max }=\lambda_{0}^{2} / 4 \delta \lambda$, where $\delta \lambda$ is the spectral resolution and $\lambda_{0}$ is the centre wavelength of the spectrum. For our current setup, $\lambda_{0}=745 \mathrm{~nm}$ and $\delta \lambda=0.52 \mathrm{~nm}$, and this results in a maximum measurable amplitude of approximately $270 \mu \mathrm{m}$. As a result, the SOCVT system is able to characterize microscale vibrations. The use of light with longer centre wavelengths and a spectrometer with higher spectral resolution will improve this maximum measurable amplitude significantly, for example, to a few millimetres.

PZT stack actuators' dynamic hysteretic behaviour is vital for active vibration control and high-precision instrumentation, especially for nanopositioning. The calibration of the actuator was performed by varying the input voltage on the entire range in the ascending and descending directions. The voltages from 0 to 100 volts, which were generated by an Agilent signal generator and were amplified by a Chroma programmable DC power supply, were applied to the Thorlabs PZT stack actuator in the ascending and descending directions. The dynamic hysteretic curves were measured by the developed SOCVT and a commercial STIL MiCROMESURE system. MICROMESURE is a modular measurement system dedicated to high resolution 3D microtopography and to shape and texture analysis. It can be used to measure profiles or surfaces of samples. The optical pen used was CL2-MG140 and the corresponding axial resolution of the MICROMESURE system was $22 \mathrm{~nm}$. If average of 10 was taken, the axial resolution of the MICROMESURE system would be $8 \mathrm{~nm}$. The measurement type "a point as function of time" was selected to measure PZT's dynamic hysteretic curve and we used 10 average to increase the axial resolution of the MICROMESURE system. Fig.3 (a) showed PZT's 
dynamic hysteretic curves measured by the two systems. It could be seen that the dynamic hysteretic curve measured by SOCVT was smooth whist the one obtained by the MICROMESURE system was noisy. This demonstrated the performance of the developed SOCVT was higher than the one of MICROMESURE system. Fig.3 (b) showed the dynamic hysteretic curves for the different ascending and descending voltage. The curves were smooth, and the displacement range was from nanoscale to micron scale. The SOCVT measures the absolute displacement of PZT surface without the requirement for both phase-shift modulation and the integration of velocity along the time axis, which are required for a laser Doppler vibrometer to obtain displacement information [22]. The displacement resolution of the system remains in ultrafine condition for both nanoscale and microscale displacement measurements. In addition, the nonlinear effect of PZT behaviour could be obtained from Fig. 3(b). Therefore, the enhanced SOCVT provides a tool for nonlinear investigation of PZT stack actuators, which is quite useful because nonlinear effects greatly affect the precision of PZT-based positioning.

For further validation of the nanopositioning capability of the PZT actuators, voltages from 0 to 5 volts were applied to the PZT stack actuator in the ascending direction and then the descending direction. The dynamic hysteretic curves for nanopositioning are shown in Fig. 4, from which the high repeatability and stability of the SOCVT system were demonstrated. Our SOCVT system is non-contact and noninvasive in nature; additionally, the maximum measurable range can be up to a few millimetres. These characteristics are the main advantages of the SOCVT system, which make the system an alternative method for the ultra-precise calibration of piezoelectric 
actuators. In the present work, the nanoscale-displacement impulsive behaviour of PZT stack actuators was also investigated based on the developed SOCVT system. As shown in Fig. 5, four DC voltages of $50 \mathrm{mV}, 200 \mathrm{mV}, 500 \mathrm{mV}$ and $1000 \mathrm{mV}$ were provided at a time of 50 seconds, which resulted in the surface of the PZT having a displacement with amplitudes of $1.1 \mathrm{~nm}, 4.5 \mathrm{~nm}, 11 \mathrm{~nm}$ and $21.5 \mathrm{~nm}$, respectively. The rise time and the settling time of the impulsive behaviour of PZT stack actuators could be obtained from the averaged impulse curves in Fig. 5. The results demonstrated that the developed SOCVT system is able to characterize the impulsive behaviour of PZT stack actuators.

\section{CONCLUSIONS}

In summary, real-time non-contact calibration of nanopositioning piezoelectric actuators via subnanoscale-displacement-resolution optical coherence vibration tomography enhanced using spectrum correction was presented. The developed enhanced OCT with a subnanoscale displacement resolution and with a maximum measurable amplitude of the vibrations or deformations of up to a few millimetres (which depends on the light source and spectral resolution of the spectrometer), could be used as a tool for characterizing the dynamic hysteretic behaviour, nonlinear effect and impulsive behaviour of PZT stack actuators in both nanoscale and microscale displacement ranges. SOCVT is an absolute displacement measurement method that does not require the use of phase-shift modulation and the integration of velocity along the time axis, both of which are required for a laser Doppler vibrometer to obtain the displacement information; as a result, SOCVT is attractive for application in real-time 
and in situ non-contact calibration of piezoelectric actuators that are widely used in active vibration control and high-precision equipment, especially in nanopositioning.

\section{ACKNOWLEDGEMENTS}

We gratefully acknowledge support from the Fujian Provincial Excellent Young Scientist Fund (2014J07007), the Chancellor's Fellowship of University of Strathclyde, the National Natural Science Foundation of China (51005077), the Education Department of Fujian Province (JA13180), the Training Program of Fujian Excellent Talents in Universities, the Specialised Research Fund for the Doctoral Program of Higher Education, the Ministry of Education, P. R. China (20133514110008) and the Ministry of Health, P.R. China (WKJ-FJ-27).

\section{NOMENCLATURE}

$I(\lambda, t) \quad$ the spectral interferogram of the interferometer of an optical coherence tomography system

$\lambda \quad$ the wavelength of light in air

$I_{0}(\lambda) \quad$ the intensity of the incident light

$\varepsilon_{0} \quad$ the vacuum permittivity

$\mu_{0} \quad$ the vacuum permeability 


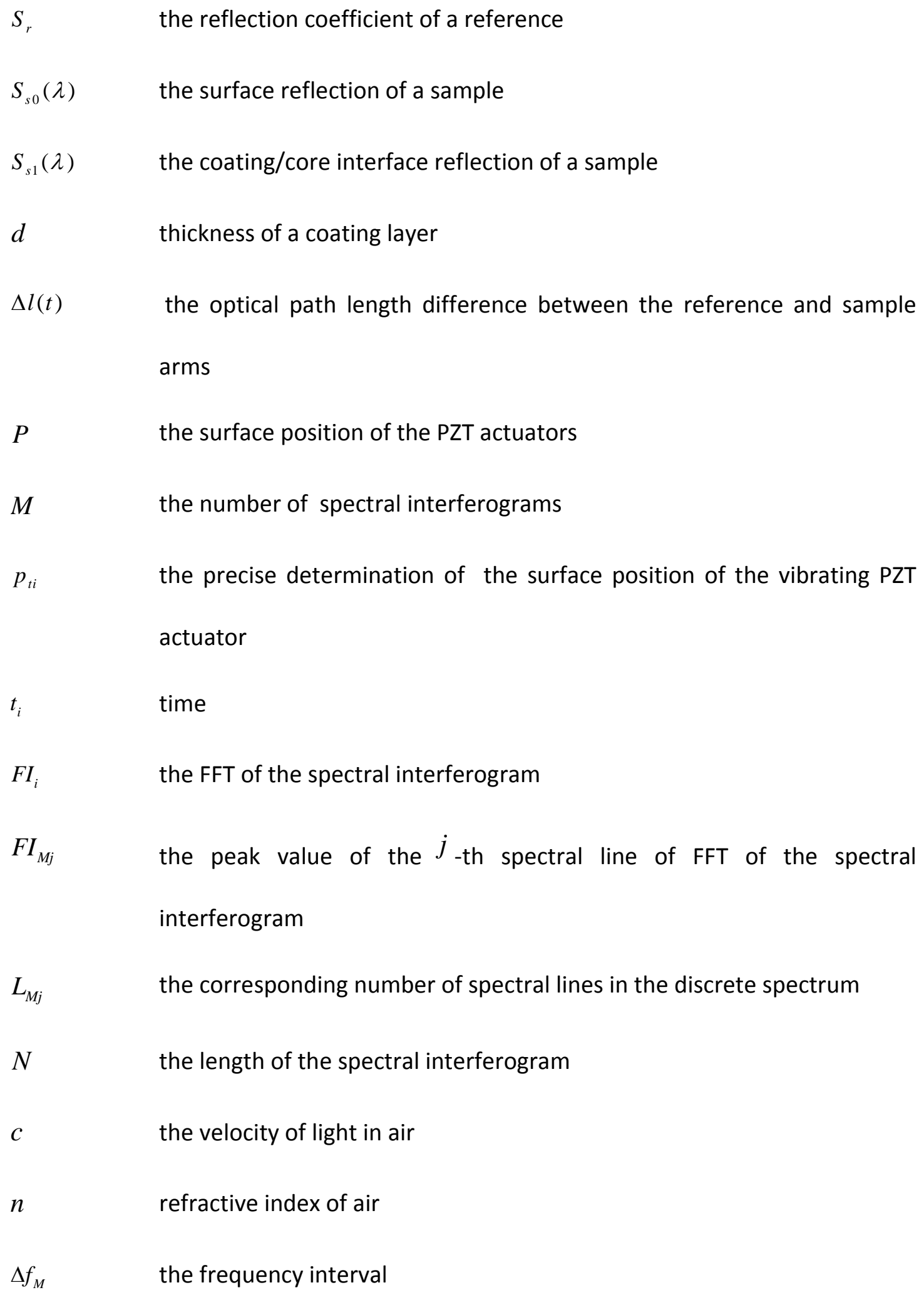


the number of spectral lines used for spectral correction 


\section{REFERENCES}

[1] Maurer L., and Leuenberger H., 2009, "Terahertz pulsed imaging and near infrared imaging to monitor the coating process of pharmaceutical tablets," International Journal of Pharmaceutics 370, pp 8-16.

[2] Leitgeb R. A., Hitenberger C.K. and Fercher A.F., 2003, "Performance of Fouier domain vs. time domain optical coherence tomography", Optics Express, 11, pp.889-894.

[3] Bashkansky M., Lewis III D., Pujari V., Reintjes J. and Yu H., 2001, "Subsurface detection and characterization of Hertzian cracks in Si3N4 balls using optical coherence tomography", NDT\&E International, 34, pp.547-555.

[4]Jonathan E., 2008, "In vivo sweat film layer thickness measured with Fourier-domain optical coherence tomography (FD-OCT)", Optics and Lasers in Engineering, 46, pp.424427.

[5] Zhong S., Shen Y., Ho L., May R. K., Zeitler J. A., Evans M., Taday P. F., Pepper M., Rades T., Gordon K. C., Muller R., and Kleinebudde P., 2011, "Non-destructive quantification of pharmaceutical tablet coatings using terahertz pulsed imaging and optical coherence tomography", Optics and Lasers in Engineering, 49(3), pp.361-365.

[6] Chen L., Zeitler J. A., Dong Y. and Shen Y., 2014, "Nondestructive evaluation of Polymer Coating Structures on Pharmaceutical Pellets using Full Field Optical Coherence Tomography", Journal of Pharmaceutical Sciences, 103, pp.161-166.

[7] Wang R., Kirkpatrick S., and Hinds M., 2007, "Phase-sensitive optical coherence elastography for mapping tissue microstrains in real time", Applied Physics Letter, 90, pp.164105 - 164105-3.

[8] Liang X., Oldenburg A., Crecea V., and Chaney E., 2008, “Optical micro-scale mapping of dynamic biomechanical tissue properties”, Optics Express, 16(15), pp.11052-11065.

[9] Chen F., Zha D., Fridberger A., Zheng J., Choudhury N., Jacques S. L., Wang R., Shi X. and Nuttall A. L., 2011, Natural Neuroscience, 14, 770.

[10] Targowski P., Gora M., Bajraszewskl T., Szkulmowskl M., Rouba B., LekawaWyslouch T., Tymlnska-wldmer L., 2006, Laser Chemistry, 2006, 1.

[11] Zhong S., Shen H., and Shen Y., 2011, "Real-time monitoring of structural vibration using spectral-domain optical coherence tomography", Optics and Lasers in Engineering 49(1), pp.127 - 131. 
[12] Stanbridge A. B., and Ewins D.J., 1999, "Modal testing using a scanning laser Doppler vibrometre", Mechanical Systems and Signal Processing, 13(2), pp.255-270.

[13] Dauksevicius R., bubulis A., Jurenas V., Ostasevicius V., and Tamulevicius S., 2004, "Investigation of dynamics of cantilever-type microstructure by laser Doppler vibrometry", Ultragarsas, 3, pp.29-32.

[14] Verrier N. and Atlan M., 2013, "Absolute measurement of small-amplitude vibrations by time-averaged heterodyne holography with a dual local oscillator", Optics Letter, 38(5), pp.739-741.

[15] Picart P., Leval J., Pascal J. C., 2005, "2D full field vibration analysis with multiplexed digital holograms", Optics Express, 13(22), pp.8882-8892.

[16] Kokkonen K., Kaivola M., 2008, "Scanning heterodyne laser interferometer for phase-sensitive absolute-amplitude measurements of surface vibrations", Applied Physics Letter 92, pp. 063502.

[17] Dhanaseka B., Ramamoorthy B., 2008, "Digital speckle interferometry for assessment of surface roughness", Optics and Lasers in Engineering, 46, pp.272-280.

[18] Zhong. J., Zhong S., Zhang Q. and Yao L., 2015, "Two-dimensional optical coherence tomography for real-time structural dynamical characterization", Optics and Lasers in Engineering, 66, pp.74-79.

[19] Grandke T., 1983, "Interpolation algorithms for discrete Fourier transforms of weighted signals", IEEE Transaction on Instrumentation and Measurement, 32(2), pp.350-355

[20] Ding K., Xie M. and Zhang X., 2000, "Phase difference correction method for phase and frequency in spectral analysis", Mechanical Systems and Signal Processing, 14(5), pp.835-843.

[21] Ding K., Cao D. and Li W., 2006, "An Approach To Discrete Spectrum Correction Based on Energy Centroid", Key Engineering Material, 321, pp.1270-1273.

[22] Kageyama K., Kimpara I., Suzuki T., Ohsawa I., Murayama H., Ito K., 1998, "Smart marine structures: an approach to the monitoring of ship structures with fibre optic sensors", Smart Materials and Structures, 7, pp.472-478. 


\section{Figure Captions List}

Fig. 1 Experimental configuration of optical coherence vibration tomography: THL, Tungsten halogen lamp; OF, Optical fibre; EP, Entrance pupil; BS, Beam-splitter; RM, Reference; WG, Waveform generator; PDCP, Programmable DC power supply; SP, Spectrometer; PC, Computer; PA, PZT stack actuator.

Fig. 2 (a) The solid lines are the displacements of sine, sawtooth, and square waves displacements without enhancement using the energy-centroid discrete spectrum correction method; subnanoscale simulated enhanced results: sine wave displacement (dash dot dot line), sawtooth wave displacement (dash point line), and triangular wave displacement (dashed line). (b) Comparisons of the displacement measurement using SOCVT with (solid blue line) and without (dashed red line) enhancement. (c) Subnanoscale vibration measurement by the developed SOCVT system. A PZT stack actuator is vibrating at $1 \mathrm{~Hz}$ with amplitudes of 4.5 $\mathrm{nm}(\mathrm{A}), 0.9 \mathrm{~nm}(\mathrm{~B})$ and $0.2 \mathrm{~nm}(\mathrm{C})$. For clarity, all of the curves are offset vertically; curves $B$ and $C$ are enlarged by a factor of 4 and 10 , respectively.

Fig. 3 The calibration of a PZT stack actuator was performed by varying the input voltage from 0 to 100 volts in both the ascending and descending directions. a) The calibration curves obtained by the developed SOCVT 
(solid line) and a commercial STIL MiCROMESURE system (dashed line);

b) the calibration curves obtained by the SOCVT could be used for characterizing the piezoelectric actuator dynamic hysteretic behaviour. The inset is the magnified view of the calibration curves for the input voltage range from 0 to 20 volts and the displacement range of $500 \mathrm{~nm}$.

Fig. 4 The calibration of a PZT stack actuator performed by varying the input voltage from 0 to 5 volts in both the ascending and descending directions. The inset is the magnified view for the input voltage below 1 volt and the displacements smaller than $20 \mathrm{~nm}$.

Fig. 5 Nanoscale-displacement impulse test of a PZT stack actuator using the developed SOCVT system: original impulse curves (blue dot lines) and averaged impulse curves (red solid lines). 
Figures

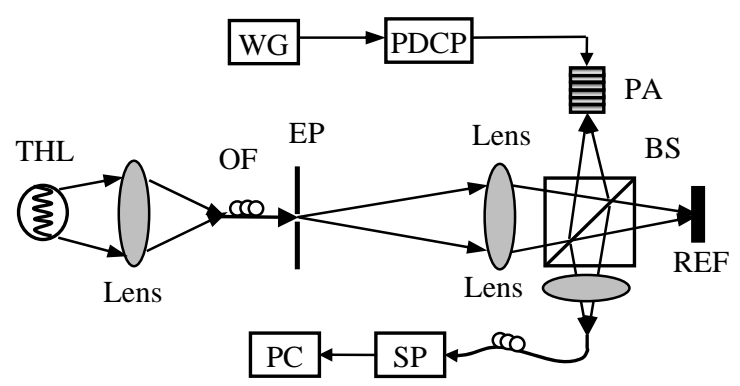

Fig. 1 

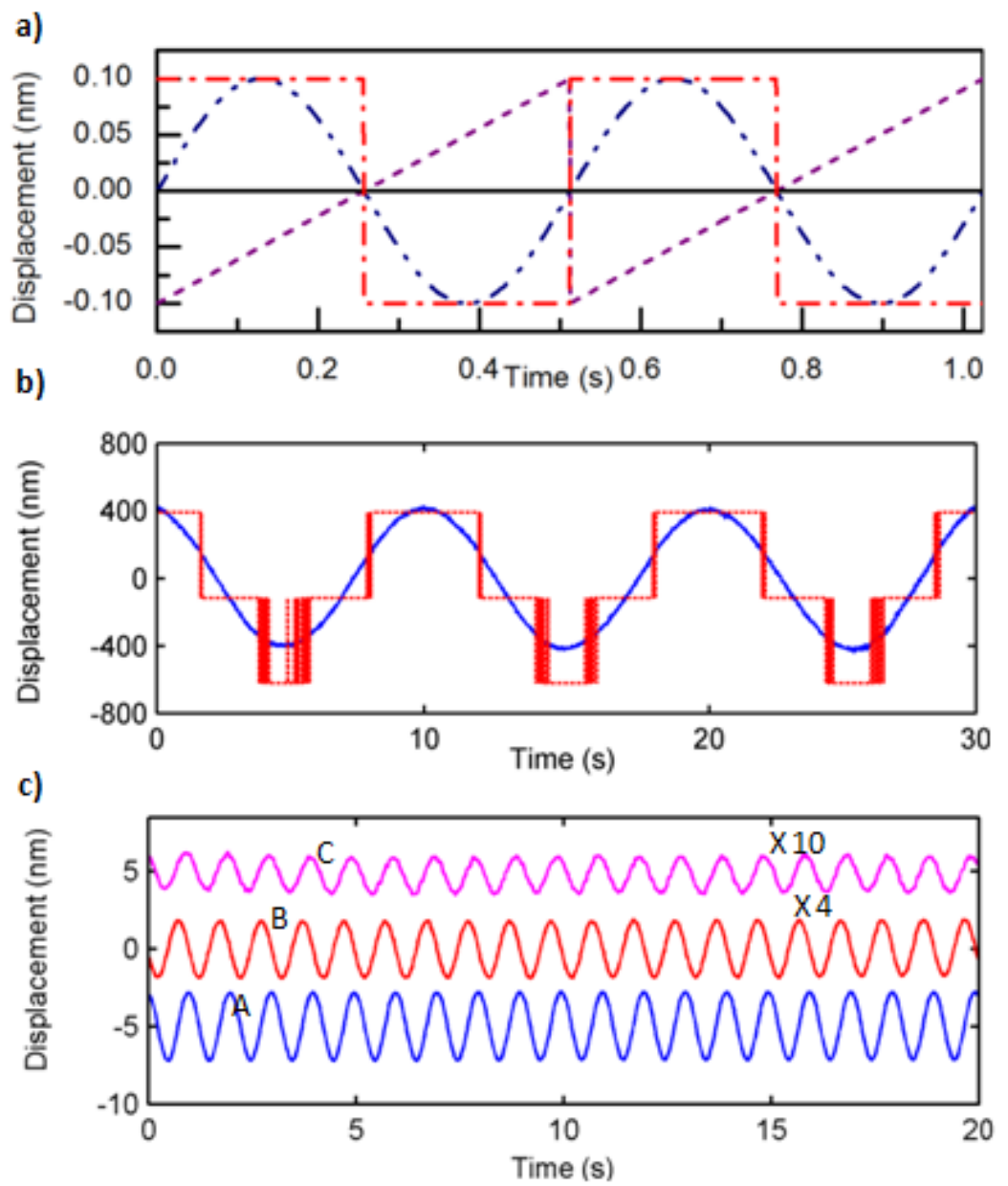

Fig. 2 

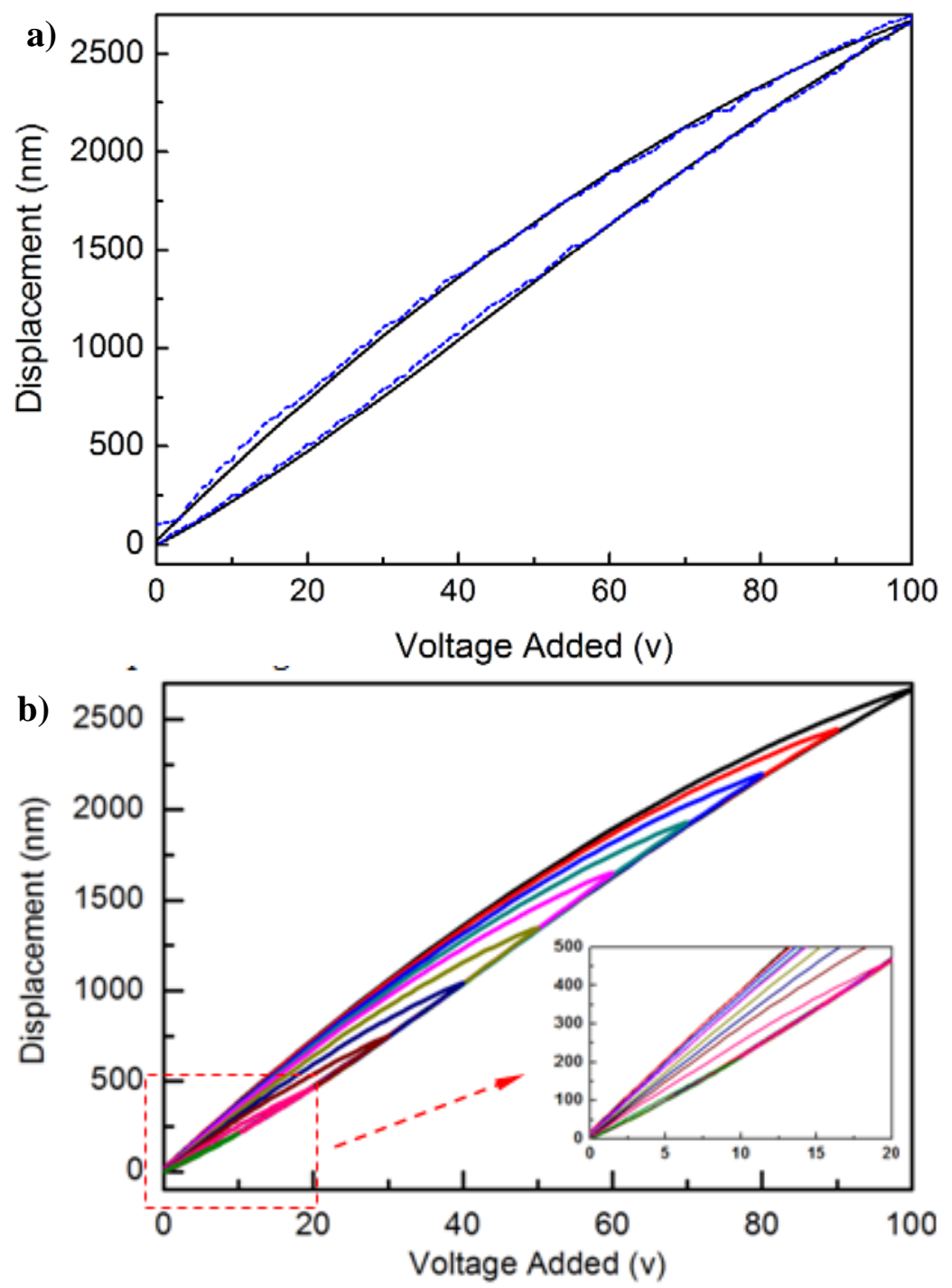

Fig. 3 


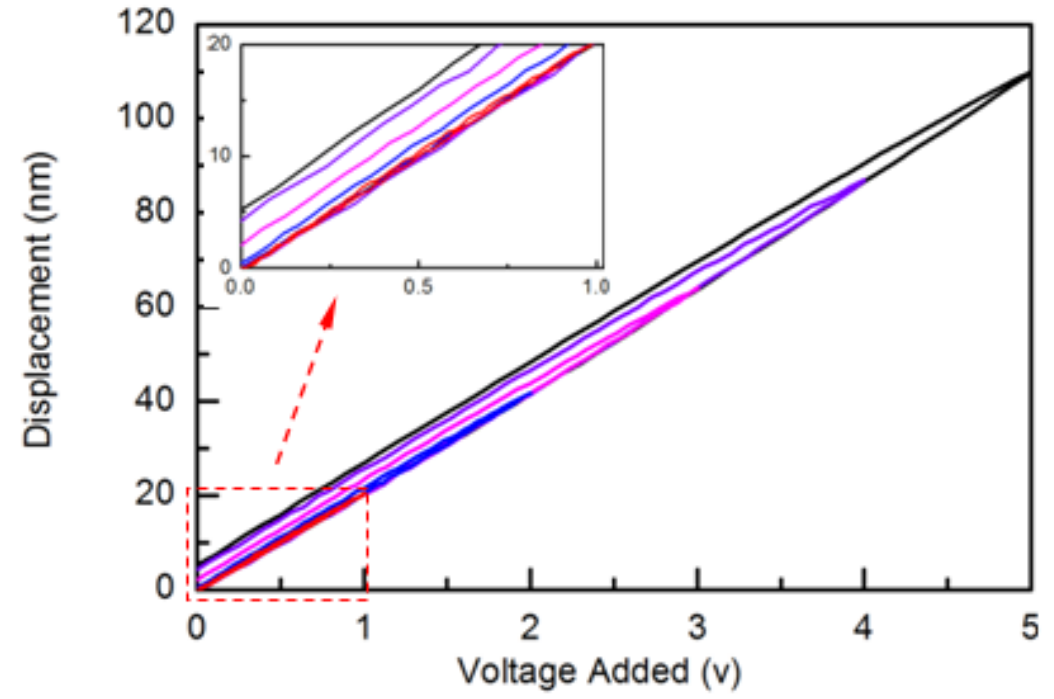

Fig. 4 


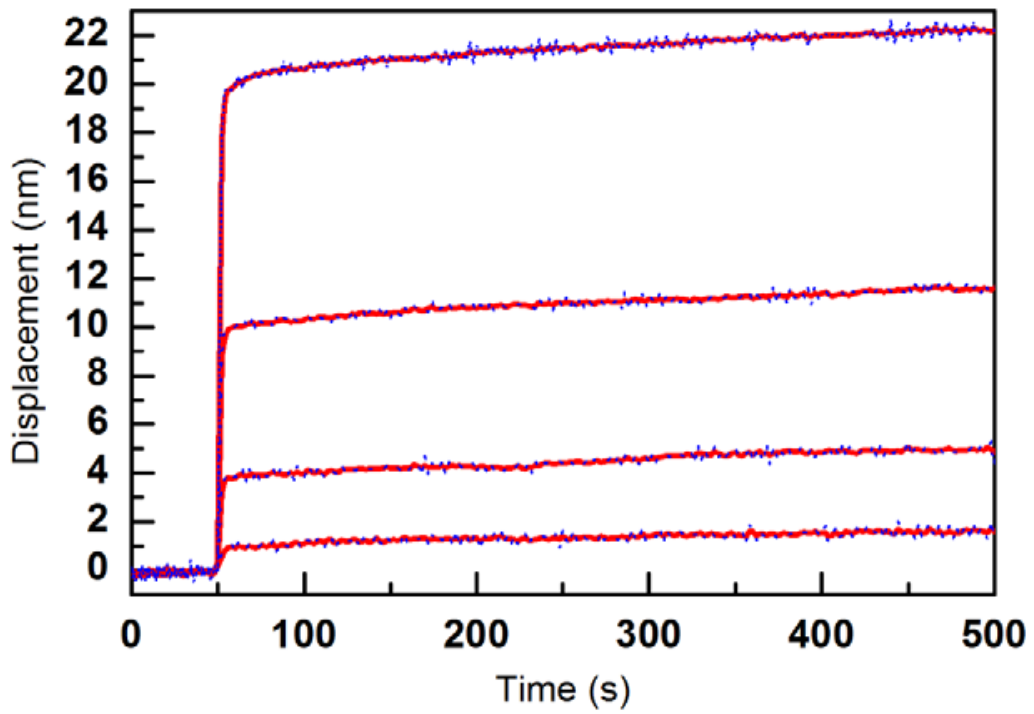

Fig. 5 